\title{
Contents, Vol. 4, 1897
}

Dermatologisehe Zeitsehrift.

Herausgegeben

Prof. Dr. O. LASSAR.

Band IV. 1897.

S. .if K. $3 / 8$ “

Berlin 1897.

VERLAG VON S. KARGER

Karlstrasse 15.

* Alle Rechte vorbehalten.

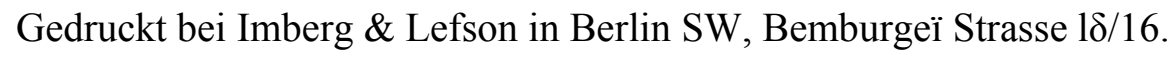

Inbalts-¥erz- $\beta$ ichn-is.

HEFT 1.

Seit» Die chirurgische Behandlung des Lupus vul-

garis. Von Dr. C. Popper in $\Lambda$ Vien

Ueber Haarfarbe und Haarfärbung. Von Dr. Paul

Richter in Berlin

34

Zur Casuistik der Lepra. Von Dr. Alfred Arnhehn 44 Zur Casuistik der Mykosis fungoides. Von Dr.

M. Jacobi in Berlin 46

Ueber seltene Formen der Quecksilber-Ver-

giftung. Von Dr. August Bos sard in Zug . . 5Ö Verhandlungen der dermatologischen Gesellschaft in Moskau. Sitzung vom 21. Februar,

30.März, 24. April, 9, October und 6. November 189697 Berliner dermatologische Gesellschaft.

Sitzung

vom 3. November 1896

114

Periodische Litteratur 124

Buc.hanzeigen 141

Notizen

147

HEFT 2.

Die Lepra in Holland und seinen Colonien. Von Dr. T. Broes van Dort in Rotterdam . . . 151

Laryngitis leprosa. Von Prof. Ramon de la Sota

y Lastra in Sevilla 163-

Die chirurgische Behandlung des Lupus- vul-garis. Von’Dr. C. Popper in Ẃien. (Schluss) . 188

$-\mathrm{IV}-$

Seite

IX. Zu $\gamma$ Casuistík der Meningomyelitis syphilitica.

Von Dr. C. Ellin gen 247 
Berliner dermatologische Gesellschaft. Sitzungen tom 1. December 1896, 5. Januar, 2. Februar und

2. März 1897264

Periodische Litteratur.319

Buchanzeigen . $\quad 330$

Notizen $\quad 333$

HEFT 3.

X. Einige epitheliale Gebilde in neuer Auffassung. Beiträge zur Pigmentfrage. Von Dr. Ernst

Kromayer in Halle 335

(Hierzu Tafel I-IV und 13 Abbildungen.)

XL Ueber Quellsalzseifen und die Anwendung der-selbenbeiHautkrankheiten. Von $\Omega$ r. E.

Bardac $\AA$

in Bad Kreuznach $\quad 400$

XII. Seltene Lokalisation yon luetischem Primär-affekt der Nasenschleimhaut. Von Dr. Jacobi in Köøigsberg 407

Berliner dermatologische Gesellschaft. Sitzuńg

tom 4. Mai $1897 \quad 410$

Periodische Litteratur 421

Henry Feulard $\dagger \quad, \cdots 427$

VIII. Section des XII. internationalen medi-

cinischen Congresses in Moskan vom 19. - 26.

August 1897. Vorläufiges Programm 428

HEFT 4.

XIII. Ueber das Chloralexantliem. Von Dr. Chrisiian Gßill

zu Aarhus in Dänemark

431

XIV. Ueber die bei Tabes dorsalis auftretenden syphi-

litischen und troponeurotischen Veränderungen der Haut und anderer Organe. Von Dr. S. Kaliscker in Berlin 443

XV. Anatomie und Pathogenese der Pemphigus-

blasen. Von Dr. Ernst Kromayer in Halle a. S. 475 (Mit 4 Abbildungen.)

XVI. Eine seltene Deformität der Fingernägel (Koi-

lonychia). Von Dr. Julius Heller in Charlottenburg-

. Berlin. (Mit 1 Abbildung) 488

XVII. Ueber vorläufige Resultate mit dem Koch'schen

Neu-Tuberkulin. Von Prof. Dr. O. Lassar . . 491

$-\mathrm{V}$

Seite

Berliner dermatolo gische Gesellschaft. Sitzung

vorn 1. Juni 1897

494

Französische Gesellschaft für Derinatologie und Syphiligraphie. Sitzung vom 20. Mai, 3. und

10. Juni $18 U ̈ 7 \ldots, \ldots \quad 504$

Periodische Litteratur 521

Yerrugas $\quad 536$

Notizen $\quad 537$

HEFT 5.

XVIII. Ueber Argentamin und Argonin. Von Dr. Rudolf 
Krösing in Stettin 53!)

XIX. Ueber das Difluordiplienyl als Wundheilmittel, speciell in der derinatologisclieu Praxis.

$\Lambda$ 'on

Dr. P. TiltMfn in Leipzig 552

XX. Ueber die Behandlung der venerischen Ge-

schwüre rnit Itrol (Argentum citricum purissi-

murn). Von Dr. O. VVerler in Berlin (llierzu Taf. V.) 556

XXI. Die Lepra in der ho 11 an disc hen Kolonie Surinam,

einst und jetzt. Von Dr. T. BrocS van Dort in

Rotterdam . . 591

XXII. ZurKenntnis des Leucoderma syphiliticum. Von

Dr. J. V. Hjeltnan in Björneborg . . . . . 606

XXIII. Zur Casuistik der trophischen Erkrankungen

der llaut. Von Dr. Bogumil W. Beroii in Sofia.

(Mit 2 Abbildungen) 614

XXIV. Zwei Fälle von Dermatitis herpetiformis (Düh-

ring). Von Dr. V. Allgcyer in Turin. (M. 1 Abbildg.) 622

Ansprache bei Bröffnung der dermatologischen Section des XII. internat. med. Congresses in

Moskau. Gehalten $\tau$ on 0. Lassar 632

Atti della Società Italiana di Dermatologia e Sifilografia in Rom vom 18. - 21. October 1885636

Periodische Litteratur 646

Buchanzeigen 664

Der XII. Internationale medicinische Congress

zu Moskau. VIII. Section 666

HEFT 6.

XXV. Untersuchun ge $\cdot n$ fiber das Vorkommen der eosi-nophilen Zellen im Blut bei Syphilis und Hautkrankheiten. Von Dr. W. Peter in Königsberg 669

$\gamma{ }_{-}$

Seite-

XXVI. Ueber Sclerodermie. Von San.-Rath Dr. Wagner

in Ribnitz (Mit 2 Abbildgn.) 6 92"

XXVII. Zur Kenntnis des Leucoderma syphiliticum.

Von Dr. J. V. Hjelman in Björneborg. (Schluss) . fi9( > XXVIII. Casuistisches. Von Dr. E.

Kromayer in Halle a. S. 70T Berliner dermatologische Gesellschaft. Sitzung

yom 6. Juli .

Der XII. Internationale medicinische Congress

in Moskau vom 19. -20. August 1897 .... 721 69. Versammlung deutscher Naturforscher und

Aerzte in Braunschweig 760'

Französische Gesellschaft für Dermatologie und

Syphiligraphie. Sitzung $\tau$ om 8. Juli 1897. . . 77( >

Periodische Litteratur ; . . 781

Besprechungen $\quad 818-$

Buchanzeigen . $\quad 826$

Notizen 831 